\title{
Physical characterisation of microporous and nanoporous polymer films by atomic force microscopy, scanning electron microscopy and high speed video microphotography
}

\author{
M.S. Barrow ${ }^{\mathrm{a}, *}$, R.L. Jones ${ }^{\text {a }}$, J.O. Park ${ }^{\mathrm{b}}$, M. Srinivasarao ${ }^{\mathrm{b}}$, P.R. Williams ${ }^{\mathrm{a}}$ and C.J. Wright $^{\mathrm{a}}$ \\ ${ }^{a}$ Centre for Complex Fluids Processing, School of Engineering, University of Wales Swansea, \\ Singleton Park, Swansea, SA2 8PP, UK \\ ${ }^{\mathrm{b}}$ School of Polymer, Textile and Fiber Engineering, Georgia Institute of Technology, Atlanta, \\ GA 30332, USA
}

\begin{abstract}
We report studies of ordered microporous and nanoporous polymer films formed by the evaporation of polymer solutions following exposure to a humid atmosphere. High speed microphotographic (HSMP) studies of the formation process showed that near the surface of the polymer solution, vapour condensation produced near mono-disperse water droplets which form a close-packed monolayer (or 'breath figure'). Following the evaporation of the solvent, characterisation of the solid by Atomic Force Microscopy and Scanning Electron Microscopy revealed that the surface of the polymer film is characterised by extensive regions of hexagonally close-packed microscopic pores, whose spatial arrangement replicates that of the initial droplet monolayer. Characterisation of sections of the film by Atomic Force Microscopy established that the surficial pores represent open sections of sub-surficial spheroidal cavities formed by encapsulation of the water droplets within the polymer solution. An interesting feature of the results is the occurrence of nano-scale pores at the film surface and at (and within) the walls of the sub-surficial microscopic pores. This is the first physical evidence report of such features in porous polymer films produced by a process involving breath-figures. Their dimensions suggest that more detailed structural investigations will require alternative techniques to conventional, optical methods.
\end{abstract}

Keywords: Microporous and nanoporous polymer films, condensation patterns, AFM, SEM

\section{Introduction}

The term 'breath figures' refers to the arrangement of water droplets formed by the condensation of water vapour onto either a cold solid or liquid surface [1,2]. In a humid atmosphere, the cooling associated with the evaporation of a volatile solvent from a polymer solution may be sufficient to initiate and sustain the condensation of water vapour at the solution surface, thereby forming a suspended water droplet phase. If the solution is sufficiently dense then the droplets may grow and, by a process of rearrangement at the surface of the solution, form a structural 'template' consisting of a colloidal monolayer of non-coalescing droplets [3-5].

\footnotetext{
${ }^{*}$ Corresponding author. Fax: 44 (0)1792 295676; E-mail: M.S.Barrow@ swansea.ac.uk.
} 
Several workers have reported that this template is ultimately transferred at the surface of, and within, the resulting solid polymer film in the form of ordered arrangements of microscopic pores [5]. Whereas solvents containing a concentrated population of droplets ultimately form polydisperse systems by droplet coalescence, certain combinations of polymer and solvent enable droplet coalescence to be suppressed, leading to the formation of porous films with a high degree of monodispersity, in terms of pore size distribution [5-7]. The latter feature lends the polymer films potential application in areas such as tissue culture [8] and controlled drug release [9] while their pore size (typically $0.2-10 \mu \mathrm{m}$ in diameter) makes them suitable candidates for evaluation as synthetic separation membrane products.

In general, techniques for the production of micropatterned surfaces by the lithographic translation of a pre-existing pattern or 'template' onto the surface of an appropriate substrate are well-established [10-12] but the development of convenient methods for fabricating large areas of uniform structures at the sub-micron length scale remains a challenging problem. In addressing this problem we have studied the 'breath figure'-driven templating process described above, our interest in it being stimulated by the fact that it offers the potential for a degree of dynamic control over the templating mechanism, and hence of the lengthscale of the resulting pores [5].

This is an important aspect of any potential enabling technology in areas such as Tissue Engineering, in which current fabrication methods for the production of synthetic microporous 'scaffolds' for tissue growth (i.e. a synthetic extracellular matrix) usually result in irregular pore morphologies [13-15]. The 'breath figure'-driven fabrication technique studied herein represents a possible means by which the characteristic dimensions and morphology of such 'scaffolds' may be controlled and significantly improved. However, in order to establish a basis for exploiting the 'breath figure'-driven process in the production of functional pore morphologies, it is necessary to gain an improved understanding of the production process which involves a complex (but as yet unquantified) interplay of fluid mechanical, thermal and surficial mechanisms. These include (but are not necessarily restricted to) the dynamics of non-coalescing liquid drops; Bénard-Marangoni convection; immersion capillary forces; thermocapillary-flows; shear-induced ordering; phase separation; thermosolutal flows; air flow velocity, air flow regime and ambient humidity level.

At present no satisfactory theory exists to explain the formation of the complex pore morphology within the solid polymer scaffolds formed by the breath figure process; and the evidence used to support previous explanations of the process of scaffold formation remain incomplete and (essentially) circumstantial. It is clear that there is a need for detailed studies of the pore morphology at a micron and sub-micron level of discrimination. Herein we report the use of Atomic Force Microscopy and Scanning Electron Microscopy in such studies.

\section{Experimental}

The work reported considers the microscale structures produced in monocarboxy terminated polystyrene (Scientific Polymer Products, USA, Mw $=50,000$ ). Polymer solutions were prepared by dissolving a quantity of polymer in carbon disulphide (Sigma Aldrich Chemical Co., UK) to produce polymer solutions ranging from 0.5 to $5 \mathrm{wt} \%$ of polymer. Although the use of solvents of different densities facilitates the preparation of multi-layered sub-surficial pores, the present work is primarily concerned with the production of monolayers which are located in close proximity to the surface of the sample.

The samples were prepared within a flow cell wherein a small quantity of polymer solution was cast onto a glass surface and was subsequently exposed to an airflow of regulated temperature and humidity. 


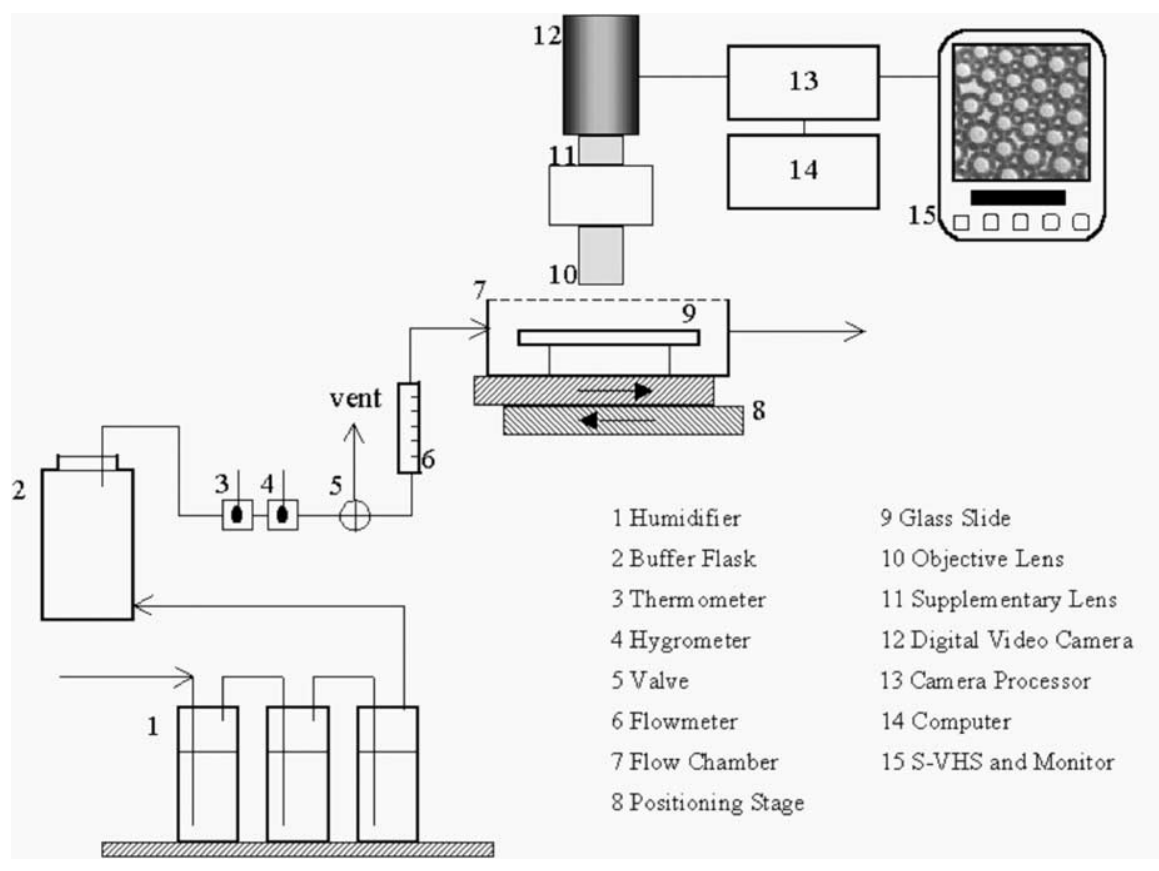

Fig. 1. Apparatus consisting of the high speed microphotographic equipment and the glycerol-water humidifier incorporating an in-line thermocouple and hygrometer.

As the sample cooled, the progressive growth and rearrangement of condensed water droplets at the sample surface was observed using a high-speed digital video camera attached to a standard microscope incorporating long working distance objective lenses.

The video system used was a Kodak Ektapro4540mx system, the camera being connected directly to a video image processor which facilitated the time-referenced sequential storage of 8-bit monochrome digital images. The system is capable of recording at up to 4500 frames per second (f.p.s) at its maximum resolution of $256 \times 256$ pixels. The camera output was also relayed through a video monitor to provide remote observation with simultaneous recording provided by an S-VHS video recorder. The complete apparatus is illustrated in Fig. 1.

In the present work, standard glass microscope slides were used as the supporting substrate. The underside of each slide was centrally inscribed with a fine 'cross-hair' and placed inside a perspex flow cell which was attached to a positioning stage. The lid of the flow cell was attached by pressure sensitive adhesive tape and contained an observation window positioned above the slide. The position of the cell and the focus of the microscope were adjusted until the 'cross-hair' reference was located. Subsequently, the microscope focal point was raised to a position slightly above the upper surface of the slide. The slide position was then fixed throughout the experiment. After purging the cell with dry compressed air, a quantity of solution was injected, through the wall of the flow cell, onto the glass slide using a syringe.

A source of compressed air was passed, at ambient temperature and known flowrate, through a series of glycerol-water solutions, the relative proportions of which were adjusted to control the humidity of the exiting flow. The humidified air was then directed through a large buffer flask containing glass wool and finally through a gas flowmeter. After the polymer solution was cast onto the glass slide, the humidified air was diverted through the flow cell and the camera was triggered. 
Following the evaporation of the solvent, the residual solid films (approximate thickness $20 \mu \mathrm{m}$ ) were inspected using an Atomic Force Microscope (AFM) and a Scanning Electron Microscope (SEM). The AFM used in the present work was a Digital Instruments Dimension ${ }^{\mathrm{TM}} 3100$ operated through a Nanoscope IV Controller and software (Veeco Metrology Group, US): the SEM was a Phillips FEI XL30CP. The principle mode of operation of the AFM in the present work was intermittent contact (tapping) mode but conventional contact mode was also employed.

The AFM was also employed to reveal additional features of the sub-surficial pore morphology in a novel manner. Although the upper layer of the polymer film could be removed in an ad hoc manner by 'peeling' it away, using a previously affixed layer of adhesive tape, more satisfactory results were achieved by systematically removing material by the action of a scanning AFM probe. In this procedure the surface was first imaged and the scanning tip was then positioned inside a surface pore, after which the AFM feedback control settings were disabled and the scan area increased. Without the application of feedback control, the scanning probe was forced into the surface structure and subsequently removed the upper surface to a depth of several microns. The subsequent attachment of a new scanning probe tip then permitted AFM imaging of the freshly exposed sub-surficial region. In this way the sub-surficial features of the films were investigated in a precise manner, with minimal disruption to the surrounding structure.

\section{Results}

As the solution surface was often subject to rapid thermoconvective flows, it was necessary to observe the behaviour of the water droplets using the HSMP system. Complementary studies of solutions within which the flows were sufficiently reduced were sometimes possible, the latter being recorded using the S-VHS system to provide extended documentation of the droplet systems. Figure 2 shows several (nonsuccessive) images from a HSMP sequence which record various stages in the growth and rearrangement of water droplets on the surface of a polymer solution.

In frame \#1, Fig. 2, isolated water droplets are recorded adjacent to small aggregates of stabilised (non-coalescing) droplets. Between frames \#1-\#4, the droplets increase in size while forming larger aggregates (often promoted by surface flows), the latter eventually forming a colloidal monolayer which represents the template for the eventual formation of the solid, porous polymer films (see Fig. 3).

Following solidification of the polymer, transmitted light microscopy suggested the presence of close packed pores, whereas the results produced by the AFM revealed an ordered, porous array characterised by a (relatively) large value of inter-pore separation (Fig. 4a). Figure 4a shows an AFM scan of a polymer surface in which the open spaced hexagonal structure may be seen, the structure consisted of circular pores of nominal pore diameter, $d_{\mathrm{p}}=3.3 \mu \mathrm{m}$, separated by a distance, $d_{\mathrm{s}}=2.6 \mu \mathrm{m}$.

The presence of sub-surficial cavities were revealed in experiments in which the surficial structure (consisting of a widely spaced hexagonal array of pores) was progressively removed using the AFM tip (see Fig. 4b). The sub-surficial structure thereby exposed was also found to exhibit a highly regular morphology, consisting of extensive regions of closely packed cells.

In the collection of the data presented in Fig. 5, a region of the surface material was removed prior to the AFM probe being positioned such that its scan range encompassed both the region from which material had been removed and that containing preserved material. The vertical distance between the remaining upper surface and the exposed sub-surficial structure (see upper right, Fig. 5) was commensurate with the maximum piezo displacement of the AFM scanner $(6 \mu \mathrm{m})$. Consequently only the uppermost parts of the hexagonal cell walls were accessible. 


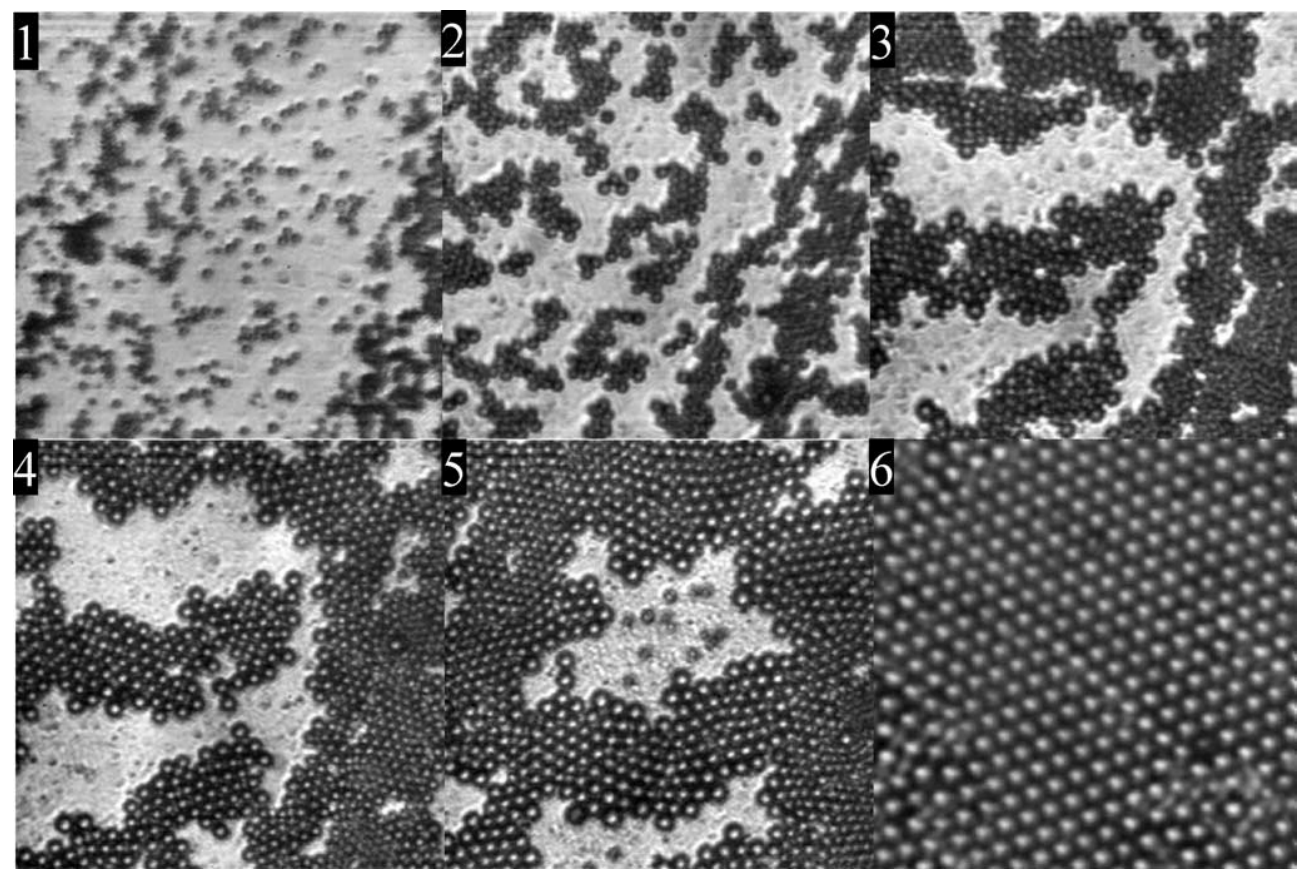

Fig. 2. Sequence of images $(180 \times 180 \mu \mathrm{m}$ square $)$ depicting the growth and aggregation of water droplets near the surface of an evaporating polymer solution. The time interval between frames \#1 and \#5 is 50 seconds. Ultimately, the droplets form an ordered hexagonal structure as shown in frame \#6.

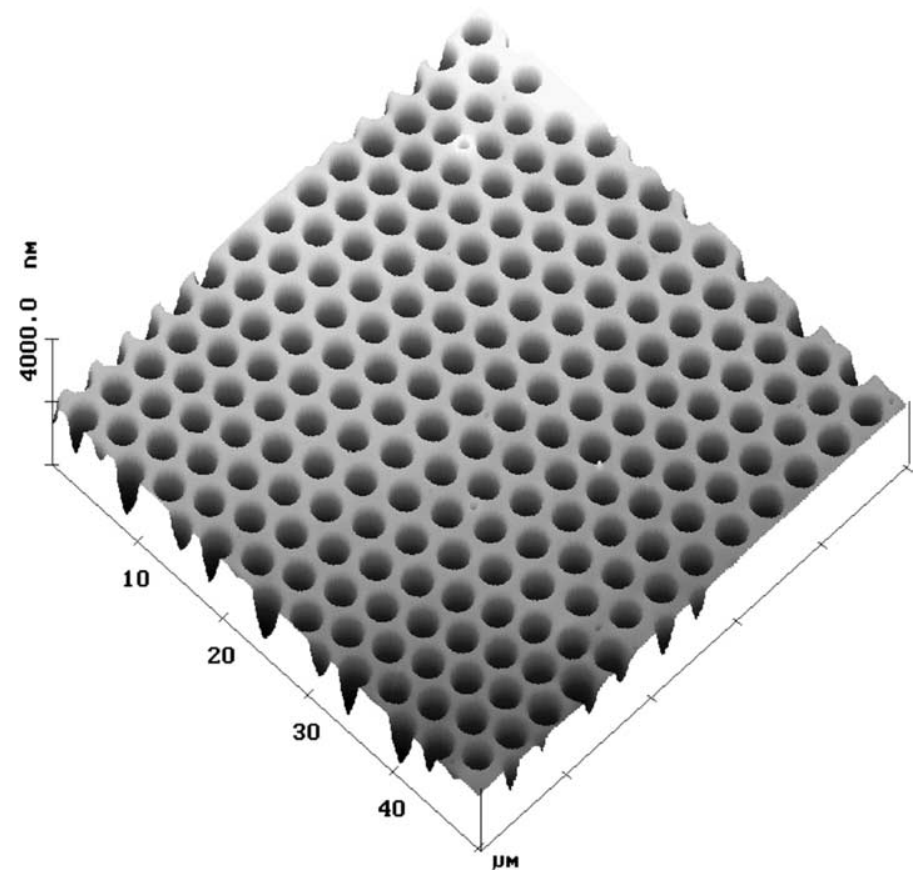

Fig. 3. AFM image of the surface of a solid polymer film revealing a hexagonal arrangement of pores produced after evaporation of both solvent and water. 


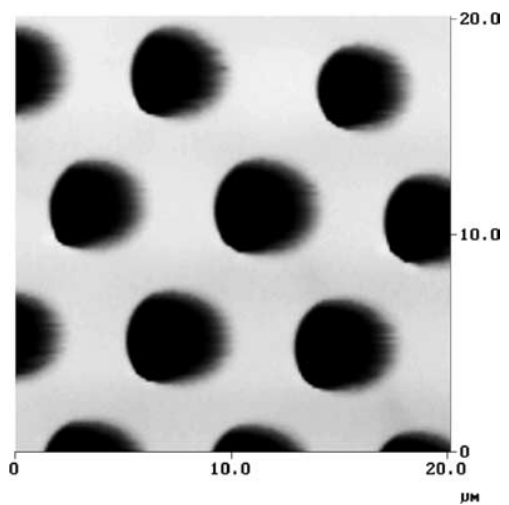

(a)

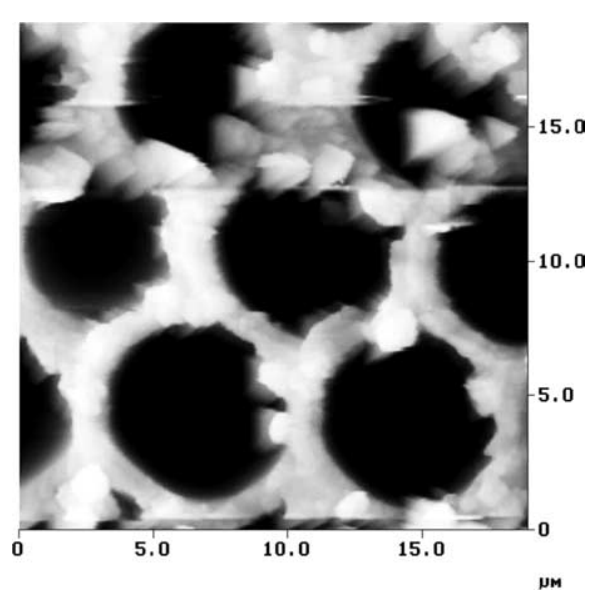

(b)

Fig. 4. AFM images of the surface structure showing widely spaced pores (a) and the corresponding sub-surface close-packed structure (b) which was exposed by removal of the surface using an AFM probe.

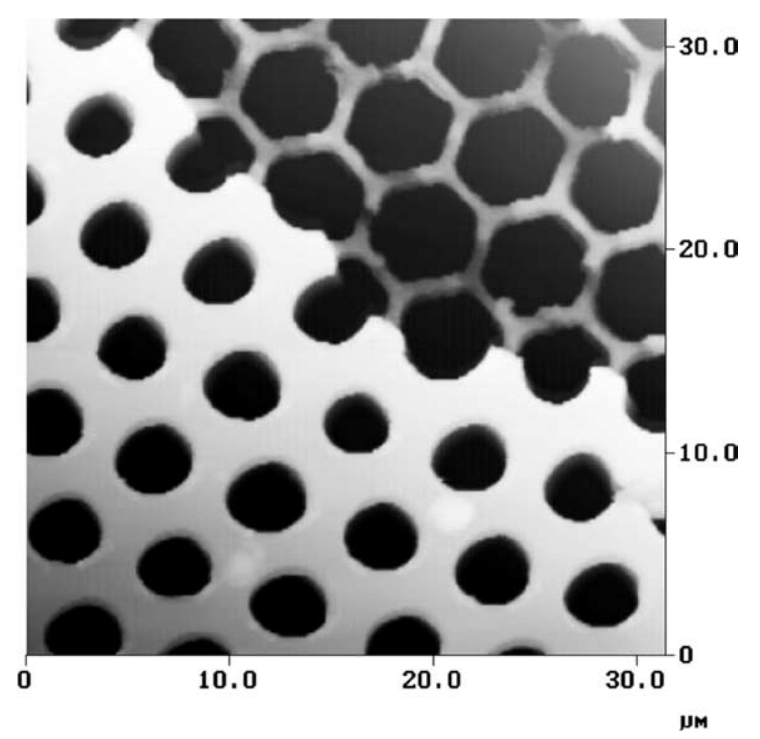

Fig. 5. AFM image of an organised mono-layer cavity structure in which the surface has been partially removed. The hexagonal cells (upper right) represent exposed sub-surficial structure.

Figures 6 and 7 show AFM and SEM results obtained in scans of the sub-surficial structures. The latter, consisting of a monolayer of micron-scale spheroidal cavities, which are located immediately beneath the corresponding surface pores. In this instance the individual cavities do not appear to be interconnected, each being separated by a layer of polymer which is several tens of nanometers in thickness. Extensive regions of the surface of the films between the micron-scale pores was found to contain a large population of nano-scale pores with a characteristic dimension of ca. $100 \mathrm{~nm}$ (see Fig. 8). The presence of the latter was hitherto unsuspected as a result of optical inspection techniques. Moreover, the results of SEM characterisation of the sub-surficial pore structure (Fig. 7) revealed the presence of nanoscopic cavities at (and within) the walls which separate the micron-scale pores. 

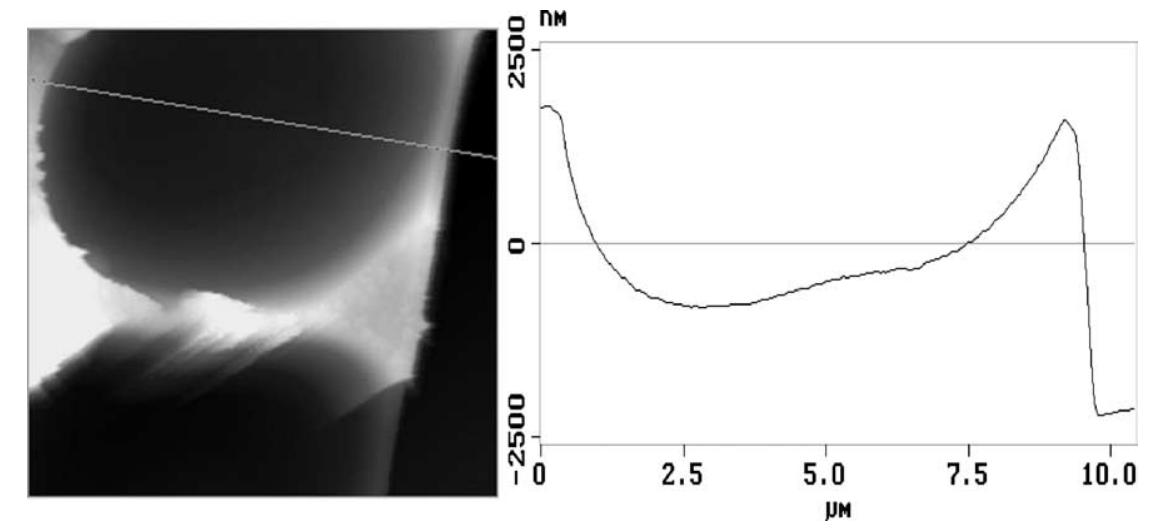

Fig. 6. An AFM image of a sectioned film showing a sub-surficial micro-cavity with cross-sectional height data. The sample was orientated so as to permit an AFM examination of the cavity walls, the film surface appears as a diagonal edge located on the far right of the image. The height data (right) corresponds to the line superimposed upon the topography image, in this instance interconnectivity between the micro-scale cavities is not apparent.

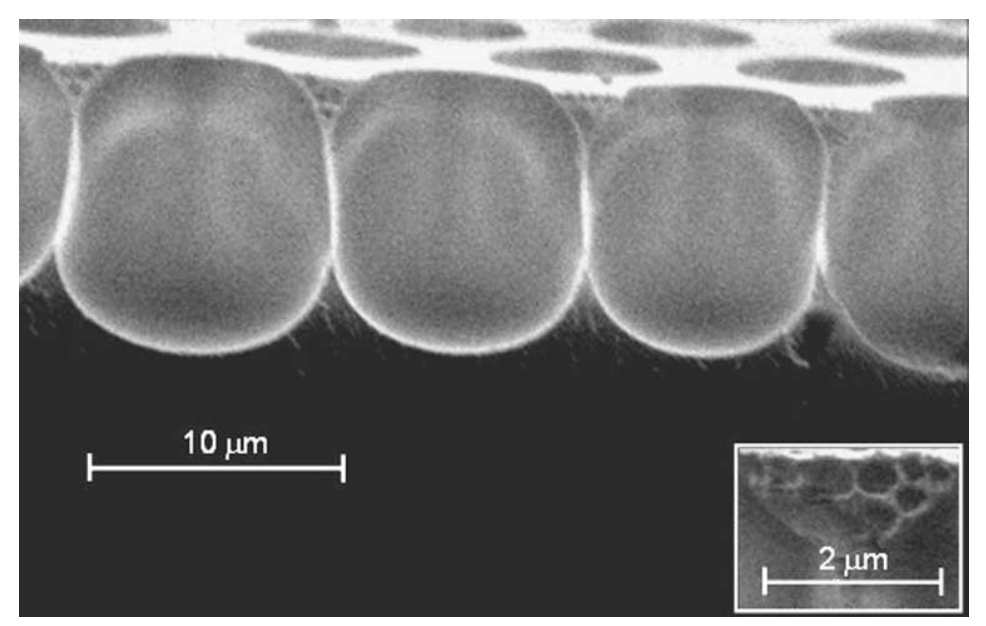

Fig. 7. SEM image of a sectioned sample showing a mono-layer of spherical sub-surficial cavities. Higher magnification studies (inset) reveal the interstitial presence of sub-micron porous structures.

\section{Discussion}

There are two notable aspects of the work reported herein. The first is the novel use of the AFM probe as a precise tool for the progressive removal of polymeric material and the exposure of underlying regions of sub-surfical pore structure for subsequent characterisation by AFM scans. In the present work this was achieved with minimal disruption to the pore structure adjacent to the scan region. The second notable aspect of this work is the finding that the film pore morphology is characterised by structure at two different lengthscales, the micron- and nanometre-scale, respectively.

It should be noted that whereas the occurrence of ordered arrays of micron-sized spheroidal subsurficial cavities and surface pores in polymer films produced by the 'breath-figure' process is well established, the extensive occurrence of nanoscopic pores within such films has not previously been reported as a result of physical characterisation techniques. (We are aware of a recent report of nanoscale 


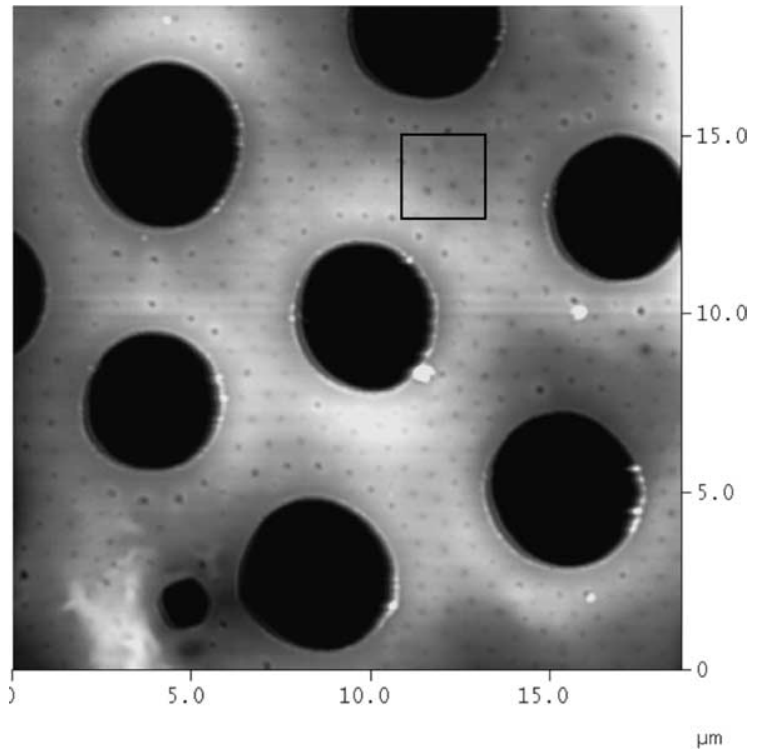

(a)

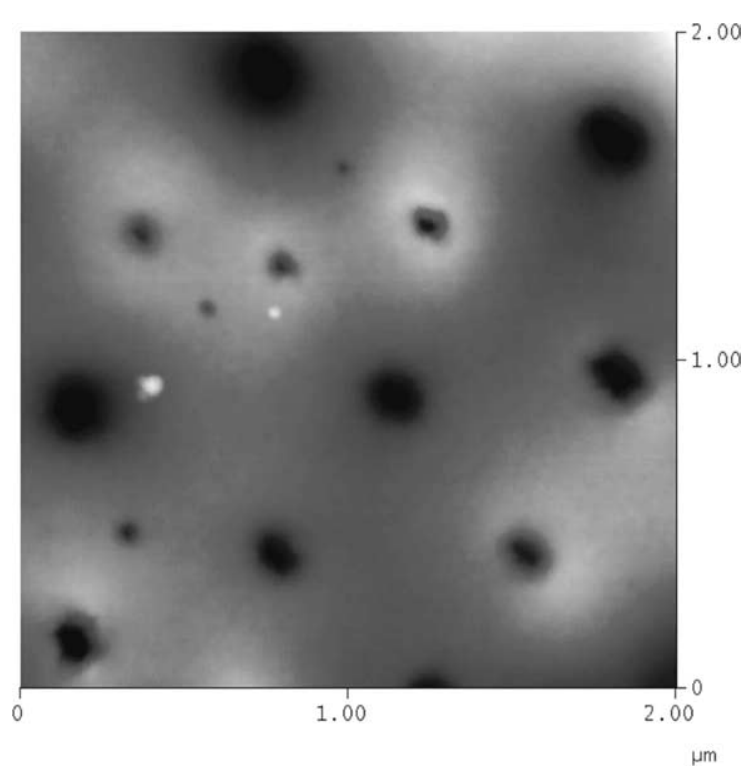

(b)

Fig. 8. (a) AFM image showing two populations of pores within the surface of the polymer film with a distinct difference in size. (b) Higher resolution image of the area marked with a square in (a).

features evident in such films from fluorescence spectra recorded by confocal microscopy [16].) This finding is particularly important in the context of the possible use of such films as biodegradable scaffolds for Tissue Engineering applications involving extracellular matrix production, or as candidate systems for drug delivery containment systems. Clearly, a failure to account for the presence of large populations of nanoscopic pores at (and within) the walls of the micron-scale pore structure will lead to significant overestimates of the mass of polymer available for biodegradation and hence of the likely lifetime of such structures in vivo. Similarly, given the potential application of porous scaffolds generated using the breath figure technique for slow release in drug delivery processes, the results of the present work suggest the presence of the smaller population of pores could have a significant influence on drug delivery timescale.

\section{Conclusions}

High speed microphotographic studies of the condensation patterns ('breath figures') formed from a humid air stream above the surface of a polymer solution containing a volatile solvent reveal the formation of a close packed colloidal array of non-coalescing water droplets. Following the evaporation of the volatile solvent, characterisation of the resulting solid surfaces by Atomic Force Microscopy and Scanning Electron Microscopy reveals that the resultant polymer film is characterised by extensive regions of hexagonally close-packed microscopic pores, whose spatial arrangement replicates that of the initial water droplet monolayer. By utilising the AFM probe to remove sections of the film's upper surface, it has been established that these pores represent open sections of a sub-surficial assembly of spheroidal cavities. In addition, nano-scale pores are formed, both at the film surface and at (and within) the walls of the microscopic sub-surficial pores. The results of this study provide the first physical 
evidence of such nano-scale features in porous polymer films produced by a process involving breathfigures, and the dimensions of these structures suggest that more detailed structural investigations will require alternative techniques to conventional, optical methods.

\section{References}

[1] D. Beysens and C.M. Knobler, Growth of breath figures, Phys. Rev. Lett. 57 (1986), 1433-1436.

[2] B.J. Briscoe and K.P Galvin, The evolution of a 2-D constrained growth system of droplets-breath figures, J. Phys. D 23 (1990), 422-428.

[3] A.V. Limaye, R.D. Narhe, A.M. Dhote and S.B. Ogale, Evidence of convective effects in breath figure formation on volatile fluid surfaces, Phys. Rev. Lett. 76 (1996), 3762-3765.

[4] O. Karthaus, N. Maruyama, X. Cieren, M. Shimomura, H. Hasegawa and T. Hashimoto, Water-assisted formation of micrometer-size honeycomb patterns of polymers, Langmuir 16 (2000), 6071-6076.

[5] M. Srinivasarao, D. Collings, A. Philips and S. Patel, Three-dimensionally ordered array of air bubbles in a polymer film, Science 292 (2001), 79-83.

[6] G. Widawski, M. Rawiso and B. Francois, Self-organized honeycomb morphology of star-polymer polystyrene films, Nature 369 (1994), 387-389.

[7] B. de Boer, U. Stalmach, H. Nijland and G. Hadziioannou, Microporous honeycomb-structured films of semiconducting block copolymers and their use as patterned templates, Adv. Mater. 12 (2000), 1581-1583.

[8] A. Folch, B. Ho, O. Beebe and M. Toner, Microfabricated elastomeric stencils for micropatterning cell cultures, J. Bio. Mat. Res. 52 (2000), 346-353.

[9] O. Pillai and R. Panchagnula, Polymers in drug delivery, Current Opinion in Chemical Biology 5 (2001), 447-451.

[10] F. Burmeister, W. Badowsky, T. Braun, S. Wieprich, J. Boneberg and P. Leiderer, Colloid monolayer lithography - A flexible approach for nanostructuring of surfaces, Applied Surface Science 144 (1999), 461-466.

[11] M. Wang and L. Zhang, Hole pattern in polymer films and its template effect on the growth of gold particles, Thin Solid Films 359 (2000), 82-87.

[12] D. Wang, T. Wu, F. Lin, J. Hou and J. Lai, A novel method for controlling the surface morphology of polymeric membranes, J. Membrane Sci. 169 (2000), 39-51.

[13] A.G. Mikos and J.S. Temenoff, Formation of highly porous biodegradable scaffolds for tissue engineering, Elec. J. Biotechnol. 3 (2000), 1-6.

[14] P.X. Ma and M.S. Ji-Won Choi, Biodegradable polymer scaffolds with well-defined interconnected spherical pore network, Tiss. Eng. 7 (2001), 23-33.

[15] K. James and J. Kohn, New biomaterials for tissue engineering, MRS Bull. 21(11) (1996), 22-27.

[16] C. Barner-Kowollik, H. Dalton, T.P. Davis and M.H. Stenzel, Nano- and micro-engineering of ordered porous blue-lightemitting films by templating well-defined organic polymers around condensing water droplets, Angew. Chem. Int. Ed. 42 (2003), 3664-3668. 


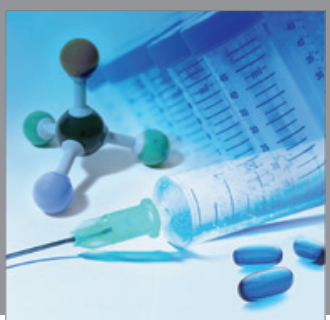

International Journal of

Medicinal Chemistry

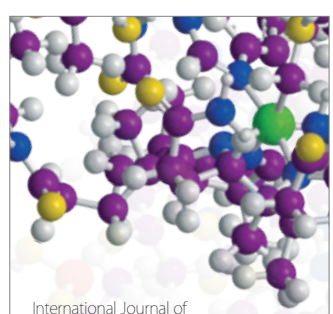

Carbohydrate Chemistry

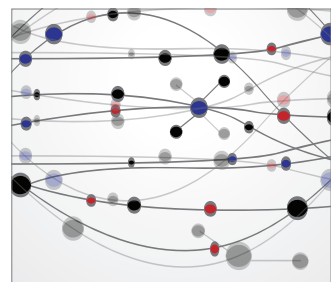

The Scientific World Journal
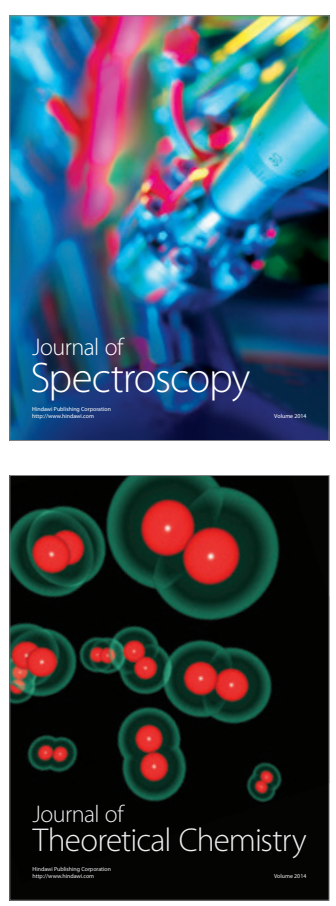
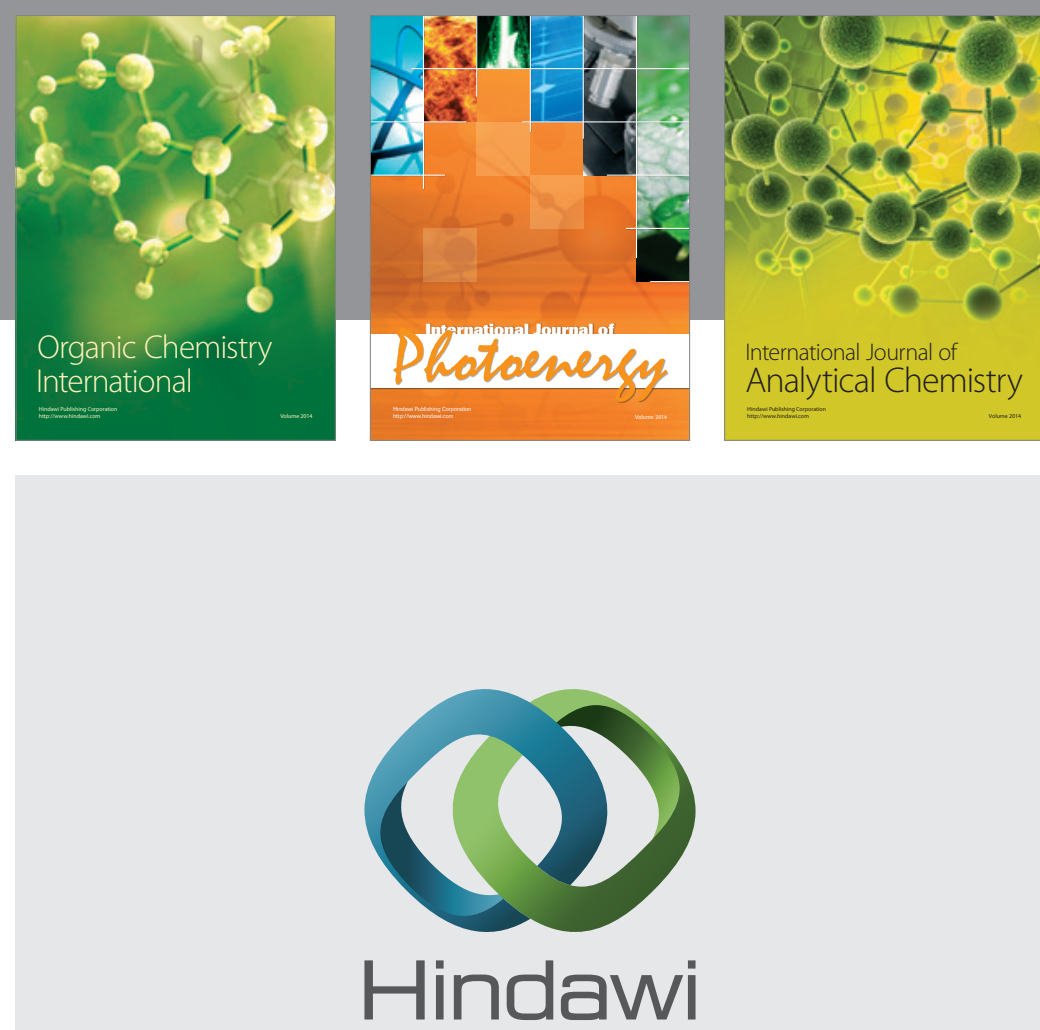

Submit your manuscripts at

http://www.hindawi.com
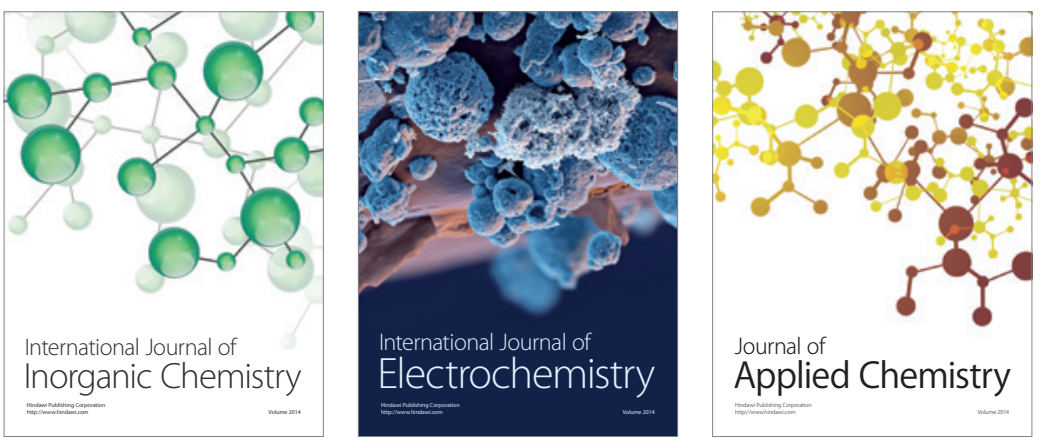

Journal of

Applied Chemistry
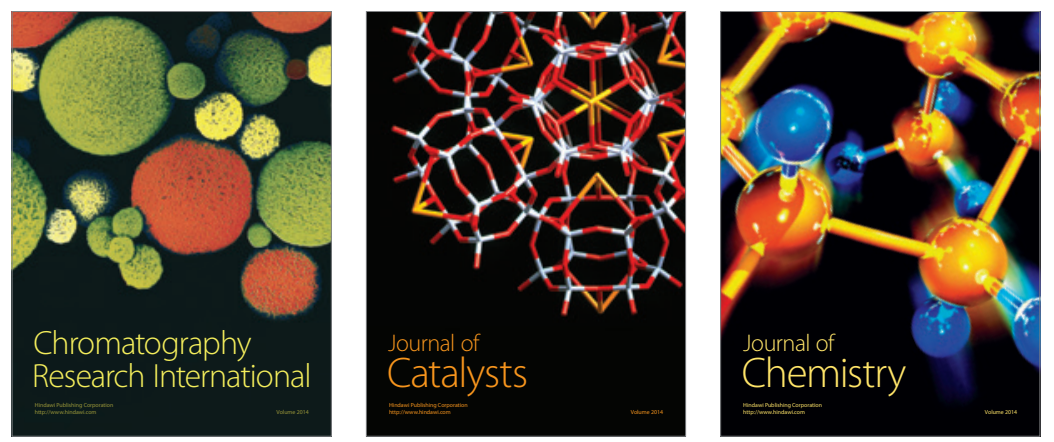
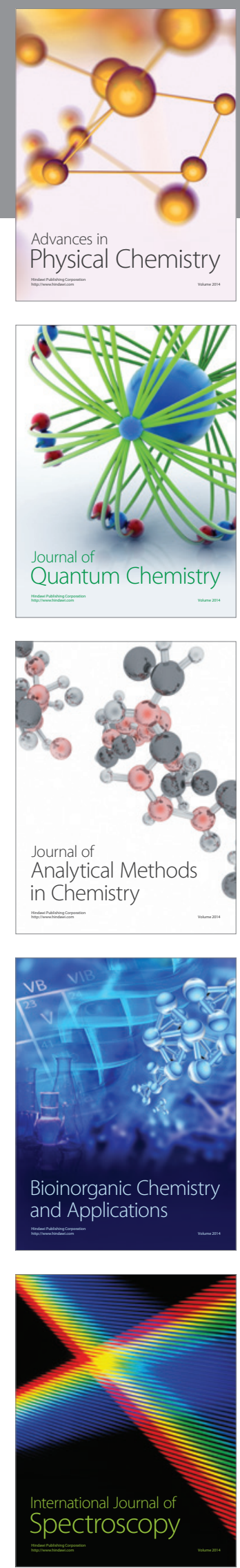